\title{
The Concept of Inductive Probability
}

\author{
Patrick Maher \\ Department of Philosophy, University of Illinois at Urbana-Champaign
}

\begin{abstract}
The word 'probability' in ordinary language has two different senses, here called inductive and physical probability. This paper examines the concept of inductive probability. Attempts to express this concept in other words are shown to be either incorrect or else trivial. In particular, inductive probability is not the same as degree of belief. It is argued that inductive probabilities exist; subjectivist arguments to the contrary are rebutted. Finally, it is argued that inductive probability is an important concept and that it is a mistake to try to replace it with the concept of degree of belief, as is usual today.
\end{abstract}

\section{Two concepts of probability}

It has often been noted that the word 'probability' is used in two different senses in ordinary language. ${ }^{1}$ In one sense, probability is relative to the available evidence and does not depend on unknown facts about the world; probability in this sense has something to do with inductive inference and so I will call it inductive probability. ${ }^{2}$ In the other sense, probability is a fact about the world and not relative to the available evidence; I will call this physical probability.

As an illustration of the difference between these two concepts, suppose you have been told that a coin is either two-headed or two-tailed but you have no information about which it is. The coin is about to be tossed. What is the probability that it will land heads? There are two natural answers to this question:

(i) $1 / 2$.

(ii) Either 0 or 1 but I do not know which.

Answer (i) is natural if the question is taken to be about inductive probability, while (ii) is the natural answer if the question is taken to be about physical probability.

Continuing with this example, suppose you now observe that the coin has a head on one side. Given the information you now have, the inductive probability of it landing heads on the next toss is 1 . Thus the inductive probability has changed with the new evidence. You also now know that the physical probability of the coin landing heads is 1 , but this probability has not changed, it was 1 before as well, only your 
knowledge about it has changed. This further illustrates how inductive probability is relative to evidence and physical probability is not.

For a different kind of illustration, consider the closing speech in Shakespeare's play Antony and Cleopatra. Cleopatra has been found dead and there is a discussion of the cause of death. Poison is considered and ruled out because it would "appear by external swelling", which is not present. It is then observed that blood is coming from a place on her breast and on her arm, and that there is a trail of the sort left by an asp snake. The play closes with a speech by Caesar that begins:

Most probable

That so she died; for her physician tells me

She hath pursued conclusions infinite

Of easy ways to die.

This is a statement of inductive probability; Caesar is saying that, given the available evidence, it is highly probable that the cause of Cleopatra's death was snake bites. It seems impossible to interpret this as a statement of physical probability; Cleopatra either died of snake bites or she did not and talk of physical probability here seems out of place. This example also illustrates the fact that inductive probabilities often do not have precise numerical values; the inductive probability in this example is high but it does not have any definite numerical value.

Although inductive and physical probability are both important concepts, the remainder of this paper will only be concerned with inductive probability.

Like many concepts of ordinary language, the concept of inductive probability is vague, and a philosopher might want to explicate it, that is, to find a more precise concept that is similar. However, before trying to explicate a concept one should clarify what it is one is explicating (Carnap, 1950, §2) and consider whether it is worth explicating; the present paper is concerned with those logically prior tasks. Hence the focus of this paper is on ordinary language, not because I am against precise explications, but because explication is blind when the explicandum is not understood.

\section{Search for a synonym}

I said that the concept of inductive probability is the evidence-relative sense that the word 'probability' has in ordinary language. It is natural to try to further clarify this concept by expressing it in other words. In this section I will examine some attempts to do that and argue that they are either incorrect or unhelpful. 


\subsection{Degree of BeLIEF}

The subjective theory of probability identifies probability with some person's degree of belief. ${ }^{3}$ This theory is now widely endorsed but it is often unclear whether it is being claimed to give a meaning that 'probability' has in ordinary language or whether it is rather claimed to be a fruitful concept that may differ from what 'probability' means in ordinary language. One example where the claim seems to be about ordinary language is Bacchus (1990, pp. 9f.), who writes that

the statement The probability that Tweety flies is greater than 0.75 is an assertion about a degree of belief; its truth is determined by the subjective state of the agent who made the statement.

It is here claimed that the statement

(1) The probability that Tweety flies is greater than 0.75 .

when uttered by person $X$ means

(2) X's degree of belief that Tweety flies is greater than 0.75 .

Let us assume that (1) is a statement of inductive probability, i.e., it is using 'probability' in the ordinary sense that is evidence-relative. I will now argue that, on this understanding, (1) when uttered by $X$ does not mean (2).

Since we are concerned with the meaning of a word in ordinary language, it is appropriate to consult a dictionary. I looked up 'probability' in four reputable dictionaries and none of them mentioned that it can mean the speaker's (or anyone else's) degree of belief. ${ }^{4}$ Also, if you ask ordinary people what 'probability' means, they will not say that it means the speaker's degree of belief. So if (1) when uttered by $X$ really meant (2), this would be a remarkable fact, since it is apparently unknown to the authors of dictionaries and competent users of the word 'probability'.

Now suppose that person $Y$ says:

(3) The probability that Tweety flies is less than 0.75 .

We would ordinarily suppose that $X$ and $Y$ have made contradictory statements, that not both (1) and (3) can be true. But if (1) when uttered by $X$ means (2) then (3) when uttered by $Y$ must mean

(4) $Y$ 's degree of belief that Tweety flies is less than 0.75 .

Statements (2) and (4) are not contradictory. So if (1) when uttered by $X$ meant (2), (1) and (3) would not be contradictory when uttered by 
different people - another remarkable discovery apparently unknown to competent users of the word 'probability'.

Suppose now that $X$ wishes to argue that (1) is indeed true. If (1) when uttered by $X$ means (2) then $X$ could prove that (1) is true by proving that (2) is true; for example, $X$ could show that he is willing to bet at odds of more than 3:1 that Tweety can fly. But we would ordinarily think that this evidence is irrelevant to the truth of (1). We ordinarily think that in order to support (1), $X$ needs to cite features of the available evidence that are relevant to whether Tweety can fly, for example, that Tweety is a bird and that most birds can fly. Thus if (1) uttered by $X$ meant (2), the sorts of arguments that could be used to support (1) would be very different to what we usually suppose.

I do not claim that the authors of dictionaries, or competent speakers of ordinary language, cannot be mistaken about the meaning of words in ordinary language, but I do claim that we should suppose them not to be mistaken unless there is a cogent argument to the contrary. Is there then any cogent argument that (1) when uttered by $X$ means (2), notwithstanding the evidence to the contrary from dictionaries and ordinary usage?

Bacchus offered no argument at all. But since Bacchus is merely following in a tradition deriving from Ramsey and de Finetti, perhaps there is an argument to be found in that tradition. Looking for such an argument, I found the following:

- De Finetti (1980, pp. 212f.; 1985, p. 353) apparently thought that a concept of probability was meaningless in the absence of some operational definition for determining its extension, that is, some procedure that could - in principle, at least - determine the values of inductive probabilities. If inductive probability were objective it would lack such an operational definition and therefore be meaningless, hence inductive probability must be subjective, a matter of some person's degree of belief.

My response: Most concepts of ordinary language do not have an operational definition but they are still meaningful. It is true that concepts lacking such a definition are apt to be vague, but this is the usual situation for concepts of ordinary language and it is not the same as meaninglessness. In addition, philosophers of science have argued at length that even the concepts of advanced sciences like physics do not have operational definitions (Suppe, 1998). And finally, we will see in Section 4.2 that degree of belief is not more amenable to operational definition than inductive probability is.

- Some statements by de Finetti could be taken to suggest that, since our assertions about inductive probabilities express our degrees of 
belief, they can have no meaning other than that we have these degrees of belief. ${ }^{5}$

My response: The conclusion does not follow from the premise. All our sincere intentional assertions express our beliefs but most such assertions are not about our beliefs. We need to distinguish between the content of an assertion and the state of mind which that assertion expresses. For example, if I say that it is raining, I am expressing my belief that it is raining but I am not asserting that I have such a belief; I am asserting that it is raining.

- Ramsey (1926) argued that objective evidence-relative probabilities do not exist, a view that de Finetti (1980, p. 199) certainly also endorsed.

My response: Even if this could be proved, it would not show that inductive probability is not an objective concept; a meaningful concept may turn out to have an empty extension, like phlogiston. I thus defer consideration of the existence of inductive probabilities to a later part of this paper.

I have thus found no cogent argument to offset the evidence of ordinary usage, which tells us that inductive probability is not the same thing as degree of belief.

\subsection{Rational DegRee of Belief}

We have just seen that inductive probability is not identifiable with actual degree of belief. I will now consider whether it is identifiable with rational degree of belief. More precisely, the view to be considered is:

(5) The inductive probability of $H$ given $E$ is the degree of belief in $H$ that is rational for a person whose total evidence is $E$.

I will now present a counterexample to (5).

Suppose that $X$ is a competitor in a sports event and knows that he will perform better if he has a high degree of belief that he will win. Then it may be rational for $X$ to have a high degree of belief that he will win, even if the inductive probability of this, given $X$ 's evidence, is low.

In this counterexample, the rationality of $X^{\prime}$ 's degree of belief depends on pragmatic considerations. To avoid such counterexamples, we might modify (5) to specify that the rationality in question is epistemic. This gives: 
(6) The inductive probability of $H$ given $E$ is the degree of belief in $H$ that is epistemically rational for a person whose total evidence is $E$.

In order to evaluate (6) we need to elucidate the concept of epistemic rationality. There are several possibilities.

A natural suggestion is that a person's beliefs are epistemically rational if they are conducive towards epistemic goals such as truth and avoidance of error. I will call this the instrumental conception of epistemic rationality. I will now argue that, on this conception, (6) is false.

Suppose (i) $X$ knows that a superior being offers tremendous knowledge to those who have a high degree of belief in $H$, (ii) $X$ 's total evidence is $E$, and (iii) the inductive probability of $H$ given $E$ is low. In view of the knowledge that is given to those who believe in $H$, it may be instrumentally epistemically rational for $X$ to have a high degree of belief in $H$, even though the inductive probability of $H$ given $E$ is low. This example is fictitious but it shows that there is a logically possible situation in which inductive probability does not coincide with degree of belief that is instrumentally epistemically rational. For concepts to be the same they need to have the same extension in all logically possible situations, so it follows that inductive probability is not the same concept as degree of belief that is instrumentally epistemically rational.

Let us then try a different conception of epistemic rationality. We might say that a person's beliefs are epistemically rational if they are supported by the person's evidence. I will call this the evidential conception of epistemic rationality.

How are we to apply this evidential conception to degrees of belief? The natural suggestion seems to be that $X$ 's degree of belief in $H$ is epistemically rational if it equals the inductive probability of $H$ given $X$ 's total evidence. It then follows that (6) is true on the evidential conception of epistemic rationality. Thus inductive probabilities can be identified with evidentially epistemically rational degrees of belief. But this is unilluminating, since 'evidentially epistemically rational degree of belief' is just an obscure term for inductive probability. ${ }^{6}$

I conclude that the identification of inductive probability with rational degree of belief does not clarify the concept of inductive probability. On some natural interpretations the identification is false and in the sense in which it is true it is trivial.

If someone tells me that they are using 'probability' to mean rational degree of belief, that does convey useful information. It tells me that they are not talking about physical probability or actual degree of belief and I may conjecture that they intend to talk about inductive 
probability. This does not contradict the conclusion I have just drawn, which may be restated this way: If someone tells me that inductive probability is the same thing as rational degree of belief then what they have said is false on some natural interpretations and is true only in a sense in which it is trivial, hence it does not clarify the concept of inductive probability.

\subsection{Justified DEGREe OF BELIEF}

We might try identifying inductive probability with justified degree of belief, rather than rational degree of belief. More precisely, the suggestion to be considered is:

(7) The inductive probability of $H$ given $E$ is the degree of belief in $H$ that a person would be justified in having if the person's total evidence is $E$.

In order to evaluate this we need to know what 'justified' means.

I will assume that we are here concerned with what is called epistemic justification as opposed, say, to pragmatic or moral justification. But what is epistemic justification? In an extensive discussion of this question, Alston (1985) distinguished two main conceptions. According to one of these, which Alston called the deontological conception, a person $X$ is epistemically justified in a belief if $X$ 's having this belief was not blameworthy from an epistemic point of view. Alston (p. 60) said that "most epistemologists who have attempted to explicate justification have set out a concept of this sort." I will now argue that if justification is taken in this deontological sense then (7) is false.

Suppose that the inductive probability of $H$ given $E$ is high. A person whose total evidence is $E$ might have a high degree of belief in $H$ but do so for some irrelevant reason - or no reason at all - and not because the person perceives any real relation between $H$ and $E$. In that case the person would deserve epistemic blame and so would not be justified (in the deontological sense) in having a high degree of belief in $H$. This is a counterexample to (7).

Conversely, a person might make a conscientious effort to determine the inductive probability of $H$ given $E$ but make some subtle error and come to the conclusion that the probability is low and as a result have a low degree of belief in $H$. We can suppose that this person is not blameworthy, in which case the person is justified (in the deontological sense) in having a low degree of belief in $H$ even though the inductive probability is high. This is another counterexample to (7).

The other main conception of justification, besides the deontological, is what Alston called the evaluative conception. On this conception, a 
person $X$ is justified in a belief if $X$ has adequate grounds for that belief. This is the same as what, in the preceding section, I called the evidential conception of epistemic rationality. I noted that on this conception, the phrase 'rational degree of belief' is just an obscure way of referring to inductive probability, and the same comment applies to 'justified degree of belief' on the evaluative conception of epistemic justification.

Thus the identification of inductive probability with justified degree of belief is either false or trivial and either way does not clarify the concept of inductive probability.

\subsection{Degree of CONFIRMATion}

Carnap $(1950, \S 9)$ took himself to be explicating a "prescientific concept of probability" that he called "probability" ; the "prescientific concept" that he had in mind seems to have been what I am calling "inductive probability". Carnap tried to clarify this concept by identifying it with "degree of confirmation". More recently, Roeper and Leblanc (1999, p. xi) interpret "the probability of $A$ as the degree of confirmation of $A$." So let us consider this view:

(8) The inductive probability of $H$ given $E$ is the degree to which $H$ is confirmed by $E$.

In order to evaluate this we need to know what 'confirmed' means here. According to Carnap (1950, preface to second edition, xviii), 'confirmation' has two senses, which I will call incremental and absolute confirmation. Something is confirmed in the incremental sense if it is made firmer or more probable; it is confirmed in the absolute sense if it is made firm or probable.

If 'confirmed' is understood in the incremental sense then (8) is false. For example, if $H$ is a simple tautology and $E$ some empirical evidence then the inductive probability of $H$ given $E$ is high but $E$ does not incrementally confirm $H$ to any degree (since the inductive probability of $H$ is just as high when $E$ is not given as when it is).

Suppose, then, that 'confirmed' is understood in the absolute sense. Since absolute confirmation is high inductive probability, it is natural to say that the degree to which $E$ absolutely confirms $H$ is the inductive probability of $H$ given $E$. With this identification, (8) is true but trivial.

Thus the identification of inductive probability with degree of confirmation is false on one natural interpretation and, in the sense in which it is true, it is trivial. Either way, the identification does not clarify the concept of inductive probability. 


\subsection{LOGICAL PROBABILITY}

In Section 2.1 we saw that inductive probability is not identifiable with a person's degree of belief; it is objective. Inductive probability is thus like the probability concept of Keynes (1921) and the "prescientific concept of probability" that Carnap (1950, p. 23) called "probability 1 ". Keynes and Carnap are regularly cited as paradigmatic advocates of "logical probability". Can we then identify inductive probability with logical probability?

That depends on what 'logical probability' means. It is often taken to mean "uniquely rational degree of belief" (Salmon, 1967, p. 68; Skyrms, 1986, p. 207; Gillies, 2000, p. 1). However, I argued in Section 2.2 that the identification of inductive probability with rational degree of belief is wrong on some natural interpretations; the only sense in which it is true is the trivial one in which 'rational degree of belief' means a degree of belief equal to the inductive probability.

Sometimes "logical probability" is characterized as a generalization of the logical consequence relation (Roeper and Leblanc, 1999, pp. xi, 142 ), which seems to mean simply that the "logical" probability of $A$ given $B$ has its maximum value if $A$ is entailed by $B$ and its minimum value if $A$ is inconsistent with $B$. However, every mathematical function that satisfies the axioms of probability is a "logical" probability in this sense, so this concept of logical probability is broader than that of inductive probability. Some authors (Franklin, 2001) take logical probability to be the one right generalization of the logical consequence relation, without saying what it means to be "right", but they have not said enough to tell us what they mean by "logical probability".

Let an elementary probability sentence be a sentence which asserts that the probability of a specific hypothesis given specific evidence has a particular value. Let a logically determinate sentence be a sentence whose truth value is determined by meanings alone, independently of empirical facts. I will say that a probability concept is logical in Carnap's sense if all elementary probability sentences for it are logically determinate. (This terminology is motivated by the characterization of logical probability in Carnap, 1950, pp. 20, 30). I think that inductive probability is logical in Carnap's sense; for example, the inductive probability that a coin will land heads when tossed, given only that the coin is either two-headed or two-tailed, does not depend on any facts about the coin, or what anybody believes, or any other empirical facts. But every mathematically defined function that satisfies the axioms of probability is also a logical probability in Carnap's sense, so the concept of logical probability in Carnap's sense is wider than the concept of inductive probability. 
We can say that inductive probability is the only probability concept in ordinary language that is logical in Carnap's sense. But this way of characterizing inductive probability differs little from my initial characterization and does not express the concept in other words.

\subsection{INDUCTIVE PROBABILITY AS PRIMITIVE}

I have now considered most of the ways in which one might try to express the concept of inductive probability in other words. I have argued that these are either incorrect or unhelpful in clarifying the concept of inductive probability. Nevertheless, this discussion has served to distinguish inductive probability from other concepts with which it is often confused.

We do not need to be able to express a concept in other words in order to understand it. We learn most concepts from examples of how the relevant words are used, not from explicit definitions. In the case of inductive probability, we have learned this concept from examples of the use of 'probability' and its cognates. I gave a few such examples in Section 1 and the interested reader could easily supply many others. I think that giving such examples is the best way to identify the concept of inductive probability.

\section{Existence of inductive probabilities}

I have argued that inductive probability is an objective concept. This will make some readers sceptical about whether inductive probabilities exist; they will suspect that the concept of inductive probability is like the concepts of phlogiston or aether in not applying to anything real. In this section I will argue that this is wrong and that inductive probabilities do exist. I will first give a positive argument for this conclusion and then respond to sceptical arguments.

\subsection{A positive ARgument}

I will assume the following existence criterion:

EC. Inductive probabilities exist if and only if there are true sentences in ordinary language which assert that a probability has a certain (precise or imprecise) value, where 'probability' is used in the inductive sense.

Someone who rejects this criterion means something different than I do by the sentence 'inductive probabilities exist'. Now consider the following sentence: 
WB. The probability that a ball is white, given that it is either white or black, is $1 / 2$.

Practically all competent speakers of ordinary language will assent to WB. ${ }^{7}$ The reference in WB to evidence, and the lack of any suggestion of a repeatable experiment, makes it clear that this is a statement of inductive probability, not physical probability. Consequently, the truth value of WB does not depend on empirical facts but is determined by the relevant concepts. So, since competent users of a language normally use the concepts of their language correctly, the fact that practically all competent users of ordinary language will assent to WB is strong evidence that WB is a true sentence of ordinary language. Hence, in the absence of any good reason to the contrary, we should conclude that WB is true, which by EC implies that inductive probabilities exist.

At this point I expect sceptics to respond that we do have "good reason to the contrary." As noted before, inductive probability is like the probability concepts of Keynes and Carnap, often called "logical probability", and these have been widely criticized. So I will now consider some of these criticisms and argue that they give no good reason to deny the existence of inductive probabilities.

\subsection{RAMSEY'S ARGUMENTS}

Ramsey motivated his own account of probability by first criticizing Keynes's theory. In a passage that continues to be cited approvingly (Gillies 2000, p. 52; Hacking 2001, p. 144), Ramsey wrote:

But let us now return to a more fundamental criticism of Mr. Keynes' views, which is the obvious one that there really do not seem to be any such things as the probability relations he describes. He supposes that, at any rate in certain cases, they can be perceived; but speaking for myself I feel confident that this is not true. I do not perceive them, and if I am to be persuaded that they exist it must be by argument; moreover, I shrewdly suspect that others do not perceive them either, because they are able to come to so very little agreement as to which of them relates any two given propositions. (1926, p. 27)

Unlike Keynes, I do not say that inductive probabilities can be "perceived", whatever that might mean. But I have argued that inductive probabilities exist and we sometimes know what they are. The passage I have just quoted from Ramsey suggests the following argument against the existence of inductive probabilities. (Here $\mathrm{P}$ is a premise and $\mathrm{C}$ is the conclusion.) 
P. People are able to come to very little agreement about inductive probabilities.

C. Inductive probabilities do not exist.

$\mathrm{P}$ is vague (what counts as "very little agreement"?) but its truth is still questionable. Ramsey himself acknowledged that "about some particular cases there is agreement" (p. 28). He asserted that "these paradoxically are always immensely complicated" but WB is a counterexample to that. In any case, whether complicated or not, there is more agreement about inductive probabilities than $\mathrm{P}$ suggests.

Ramsey continued:

If $[. .$.$] we take the simplest possible pairs of propositions such as$ "This is red" and "That is blue" or "This is red" and "That is red," whose logical relations should surely be easiest to see, no one, I think, pretends to be sure what is the probability relation which connects them. (p. 28)

I agree that nobody would pretend to be sure of a numerical value for these probabilities, but there are inequalities that most people on reflection would agree with. For example, the probability of "This is red" given "That is red" is greater than the probability of "This is red" given "That is blue". This illustrates the point that inductive probabilities are often imprecise. It does not show disagreement; it rather shows agreement, since nobody pretends to know numerical values here and practically everyone will agree on the inequalities.

Ramsey continued:

Or, perhaps, they may claim to see the relation but they will not be able to say anything about it with certainty, to state if it is more or less than $\frac{1}{3}$, or so on. They may, of course, say that it is incomparable with any numerical relation, but a relation about which so little can be truly said will be of little scientific use and it will be hard to convince a sceptic of its existence.

Although the probabilities that Ramsey is discussing lack numerical values, they are not "incomparable with any numerical relation." Since there are more than three different colors, the a priori probability of "This is red" must be less than $1 / 3$ and so its probability given "This is blue" must likewise be less than $1 / 3$. In any case, the "scientific use" of something is not relevant to whether it exists. And the question is not whether it is "hard to convince a sceptic of its existence" but whether the sceptic has any good argument to support his position; Ramsey is perhaps suggesting that vagueness provides such an argument but I have already shown that it does not.

Ramsey concluded the paragraph I have been quoting as follows: 
Besides this view is really rather paradoxical; for any believer in induction must admit that between "This is red" as conclusion and "This is round" together with a billion propositions of the form " $a$ is round and red" as evidence, there is a finite probability relation; and it is hard to suppose that as we accumulate instances there is suddenly a point, say after 233 instances, at which the probability relation becomes finite and so comparable with some numerical relations. (p. 28)

Ramsey is here attacking the view that the probability of "This is red" given "This is round" cannot be compared with any number, but that is not my view. I think the probability of "This is red" given only "This is round" is the same as the a priori probability of "This is red" and hence less than $1 / 3$. Given the additional billion propositions that Ramsey mentions, the probability of "This is red" is high, definitely greater than $1 / 2$, but it still lacks a precise numerical value. Thus the probability is always both comparable with some numbers and lacking a precise numerical value; there is no paradox here.

I have been evaluating Ramsey's apparent argument from $\mathrm{P}$ to $\mathrm{C}$. So far I have been arguing that $\mathrm{P}$ is false and responding to Ramsey's objections to indeterminate probabilities. ${ }^{8}$ Now I want to note that the argument is also invalid. Even if $\mathrm{P}$ were true, it could be that inductive probabilities exist in the (few) cases that people generally agree about. It could also be that some of the disagreement is due to some people misapplying the concept of inductive probability in cases where inductive probabilities do exist. Hence it is possible for $\mathrm{P}$ to be true and $\mathrm{C}$ false.

It may be suggested that the argument is not meant to be valid but rather is an inductive argument, the claim being merely that $\mathrm{C}$ is probable given $\mathrm{P}$. But if this is a claim about inductive probability, as it seems to be, then it is inconsistent with the conclusion $\mathrm{C}$ of the argument that it is attempting to defend.

I conclude that Ramsey's argument gives us no good reason to doubt the existence of inductive probabilities.

\subsection{Paradoxes of indifference}

I will now present another popular criticism of Keynes's conception of probability. Keynes (1921, pp. 41, 65) held that in order to quantitatively measure probabilities we need to find a set of exclusive and exhaustive alternatives that are equally probable. If there are $n$ such alternatives then the probability of each is $1 / n$ and the laws of probability can be used to derive other probabilities from these basic ones. Keynes said that a rule was needed to determine when alternatives 
are equally probable; he observed that there was a traditional rule for this purpose, which he called the "Principle of Indifference." Keynes described the principle as it was traditionally understood this way:

The Principle of Indifference asserts that if there is no known reason for predicating of our subject one rather than another of several alternatives, then relatively to such knowledge the assertions of each of these alternatives have an equal probability. (1921, p. 42)

Keynes immediately added that "this rule, as it stands, may lead to paradoxical and even contradictory conclusions" and proceeded to describe such contradictions. He then proposed a more careful formulation of this principle that, he thought, avoided the contradictions. But subsequent writers have argued that Keynes's reformulation of the principle does not prevent all contradictions (Howson and Urbach 1993, pp. 5962; Gillies 2000, pp. 37-46). More recent reformulations by Jaynes have been similarly criticized (Howson and Urbach 1993, pp. 413-417; Gillies 2000, pp. 46-48). Some philosophers take these difficulties with the Principle of Indifference to show that logical probability "does not exist" (van Fraassen, 1989, p. 292) or that "the logical interpretation ... does not allow numerical probabilities" (Gillies, 2000, p. 48).

The question I am concerned with is not whether logical probabilities, in whatever sense these authors have in mind, exist; it is whether inductive probabilities exist. Inductive probabilities are logical in Carnap's sense and it is demonstrable that logical probabilities of that kind exist since, as I noted in Section 2.5, every mathematically defined probability function is a logical probability function in Carnap's sense. It is likewise demonstrable that the existence of logical probabilities in Carnap's sense does not depend on the Principle of Indifference, since mathematically defined probability functions are defined without reference to the Principle of Indifference.

So let us now consider whether the paradoxes of indifference provide any reason to doubt the existence of that particular kind of logical probability (in Carnap's sense) that I call inductive probability. If $p$ is a stipulatively defined probability function and $p(H, E)=1 / 2$, then $p(H, E)$ has the value $1 / 2$ in virtue of the definition of $p$, not in virtue of the Principle of Indifference. Similarly, I claim that WB is true in virtue of the relevant meaning of the word 'probability' in ordinary language, not in virtue of the Principle of Indifference. The relevant meaning of 'probability' (i.e., the concept of inductive probability) is fixed and learned by examples of the use of this word, and WB is such an example; it is not learned by being given a general principle about inductive probability, such as the Principle of Indifference purports to be. Of course, once we have acquired the concept of inductive probability, we can then attempt to form general hypotheses about it, but the failure 
to find a correct general hypothesis of a certain kind cannot show that the things we are hypothesizing about do not exist. For example, failure to formulate a correct general theory of English grammar is no reason to doubt that some sentences are grammatical and some are not. Thus the difficulties with the Principle of Indifference are not a reason to doubt the existence of inductive probabilities.

\section{Importance of inductive probability}

The concept of inductive probability plays an important role in ordinary life, where it is involved in much of our reasoning and decision making. Scientists also use the concept frequently, as when they say that a hypothesis is probable given the evidence. However, most contemporary theories of probability do not concern themselves with inductive probability; instead they deal with degree of belief (also called subjective probability). Why?

Some may think that our statements about inductive probability are really statements about our degrees of belief. We saw that this is wrong in Section 2.1.

Some may think that, if our statements about inductive probability are not really about our degrees of belief, then they are not about anything that exists. We saw that this is wrong in Section 3.

I will now discuss some other reasons that seem to motivate contemporary theorists to focus on degrees of belief and put aside anything objective like inductive probability.

\subsection{Quantifiability}

The concept of inductive probability is vague and inductive probabilities often do not have numerical values. Some probability theorists regard this as a reason to study degrees of belief instead of inductive probabilities. For example de Finetti (1985, p. 359), speaking of Keynes's view that there exist "probabilities which cannot be expressed as numbers", said: "I myself regard as unacceptable, as a matter of principle, Keynes's position (the more so since the reservations which he had disappear when one adopts a subjective point of view)".

I have two responses to this. First, even if it were true that degrees of belief always have numerical values, it does not follow that we should not study inductive probability and study degree of belief instead. The concept of inductive probability plays a different role to degree of belief, so to study something else instead is to fail to understand something of importance. This is like the drunk who lost his keys and looked for 
them under the street lamp because, although he had not lost his keys there, the light was much better there.

Second, it is not true that degrees of belief are always quantifiable. On the contrary, they are also vague and often unquantifiable, just as inductive probabilities are. This is now generally admitted; even de Finetti admits that degrees of belief do not always have precise numerical values (Gillies, 2000, p. 57). Contemporary subjectivists often say that degrees of belief should be represented by intervals of numbers rather than a unique number; Howson and Urbach (1993, p. 88.) say that this proposal "corroborates Keynes's well-known thesis that degree-of-belief probabilities are only partially ordered". ${ }^{9}$ I would add that degrees of belief also often lack precise upper and lower limits, so they can't even be accurately represented by intervals of numbers.

The vagueness of inductive probability is thus not a good reason to abandon this concept in favor of degree of belief.

\subsection{Operational Definition}

Another reason that has been given for using degree of belief, rather than inductive probability, is that there is an operational definition of degree of belief whereas inductive probabilities are "entirely lacking in operational content" because they can only be known by intuition (Bernardo and Smith 1994, p. 100).

Again, I have two responses. First, even if it were true that degree of belief has an operational definition and inductive probability does not, it does not follow that we should abandon the concept of inductive probability and study degree of belief instead. As I said before, the concept of inductive probability plays a different role to degree of belief, so to study something else instead is to fail to understand something of importance. We are back with the drunkard under the street lamp.

Second, it isn't true that the concept of degree of belief has an operational definition. Degree of belief is, like inductive probability, a concept of ordinary language; the various "operational definitions" that have been proposed for degree of belief are not part of this ordinary language concept. This is confirmed by the fact that many different "operational definitions" have been proposed and they will not in general give the same result; if they were all included in the ordinary concept of degree of belief, that concept would be inconsistent, which it isn't.

The so-called "operational definitions" of degree of belief are really different explicata for the ordinary concept of degree of belief, that is, different precisely defined concepts that are meant to be similar to the ordinary concept (Carnap, 1950, $\S \S 2,3$ ). The idea that degree of belief 
has an operational definition rests on a conflation of explicandum and explicatum.

There are also explicata for inductive probability. In the approach of Carnap (1950), these consist of a probability function whose values are specified by definition for a specified domain of sentences or propositions; for a recent and accessible example, see Maher (2004). ${ }^{10}$ Hence, in the sense in which degree of belief has been operationally defined, inductive probability has also been operationally defined.

Perhaps it will be objected that explicata for inductive probability are controversial. However, there are also plenty of controversies about the best way to explicate the concept of degree of belief, as one can see from Bernardo and Smith's (sec. 2.8.3) survey of alternatives to their own explicatum.

\subsection{Justification of the AXIOMs of PROBABILITy}

Subjectivist Bayesians claim to have rigorous arguments proving that degrees of belief must satisfy the axioms of probability. As an example, I will consider the Dutch book argument presented by Gillies (pp. 5864). We first have a definition: A set of betting quotients is said to be coherent if there is no set of bets at those betting quotients that together give a sure loss. Then what Gillies calls "the Ramsey-de Finetti theorem" says: A set of betting quotients is coherent if and only if they satisfy the axioms of probability. Gillies (p. 64) says:

The Ramsey-de Finetti theorem is a remarkable achievement and clearly demonstrates the superiority of the subjective to the logical theory. Whereas in the logical theory the axioms of probability could only be justified by a vague appeal to intuition, in the subjective theory they can be proved rigorously from the eminently plausible condition of coherence.

Once again my responses are two. First, even if it is true that the axioms can be justified more convincingly for subjective than for inductive probabilities, it does not follow that we should study the former concept instead of the latter. We are again back with the drunkard under the street lamp.

Second, the axioms can not be justified with more rigor for degrees of belief than for inductive probabilities. I will show this by considering three possibilities as to what subjectivists are claiming to have rigorously proved.

1. If the claim is that actual degrees of belief have been proved to satisfy the axioms, then it is false. It is generally agreed that actual degrees of belief do not satisfy the axioms, and in any case, whether 
they do or not is an empirical question that cannot be settled by a priori arguments like Gillies gives.

2. If the claim is that rational degrees of belief have been proved to satisfy the axioms, then the proof must assume that rational degrees of belief are coherent. However, this is a very debatable assumption that has been much criticized in the literature. In particular, it has been pointed out that a person whose degrees of belief are not coherent need not be at risk of suffering a sure loss (Maher, 1993, sec. 4.6). In addition, trying to ensure that one's degrees of belief are coherent would take an inordinate amount of time and there are better ways to use the time, so rationality cannot require that one have coherent degrees of belief.

Whether or not these arguments are accepted, it must surely be admitted that the concept of rationality is vague and ambiguous. Hence the assumption, that rational degrees of belief must be coherent, can only be based on the kind of "vague appeal to intuition" that Gillies claims is not required by the subjective theory. And in my view, the assumption that inductive probabilities must be coherent, or must satisfy the axioms, is at least as plausible as the assumption that rational degrees of belief must be coherent.

3. If the claim is merely that coherent degrees of belief have been proved to satisfy the axioms, then it is true that there is a rigorous proof of this claim. However, what the proof shows is that any set of coherent betting quotients satisfies the axioms, regardless of whether those betting quotients are actual or rational degrees of belief. Thus the proof shows equally well that coherent inductive probabilities satisfy the axioms. Hence this proof, though rigorous, does not discriminate between degrees of belief and inductive probabilities.

Thus subjectivist proofs of the axioms of probability are either not rigorous or else apply equally to inductive probabilities.

\section{Conclusion}

The concept of inductive probability is the evidence-relative sense that the word 'probability' has in ordinary language. I have tried to clarify this concept by giving examples of its use and by distinguishing it from other concepts with which it is often confused. Thus I showed that inductive probability is not the same as degree of belief. It is also not the same as rational degree of belief, justified degree of belief, 
degree of confirmation, or logical probability, on at least some common ways of understanding these terms. We can, however, say that inductive probability is the concept of probability in ordinary language that is logical in Carnap's sense (that is, its elementary sentences are logically determinate).

I argued that inductive probabilities exist by arguing that there are true sentences of ordinary language that say an inductive probability has a certain value. I then discussed some popular arguments against the existence of "logical" probabilities, showing that they are not cogent arguments against the existence of inductive probabilities. The fact that people often cannot settle on a numeric value for an inductive probability merely reflects the fact that inductive probabilities often lack numeric values, it does not show that inductive probabilities don't exist. The existence of inductive probabilities depends on the relevant meaning of the word 'probability' in ordinary language, not on the Principle of Indifference, so the difficulties with that principle also do not show that inductive probabilities don't exist.

I have also argued that inductive probability is an important concept and hence worth philosophical study. It plays a major role in ordinary life and science. Also, it is not like the concept of phlogiston; it has a non-empty extension. Subjectivists have sometimes claimed that the concept of degree of belief has the advantage of being quantifiable, operationally definable, and permitting rigorous justification of the axioms of probability, but I argued that none of these claimed advantages is real. Furthermore, even if those claimed advantages were real, that would not show that inductive probability is not an important concept that is worth studying.

\section{Notes}

1 Previous discussions of these senses include Carnap (1950, ch. 2) and Hacking (1975, ch. 2).

2 Traditionally, "inductive inference" meant inference from the particular to the universal (Aristotle, Topics I 12), but today the term is often used in a wider sense to refer to any non-deductive inference (Carnap, 1950, §44B). Inductive probability relates to inductive inference in the wider sense.

3 See Kyburg and Smokler (1980) for an introduction to subjective probability and some classic papers by Ramsey, de Finetti, and others.

4 The dictionaries I consulted were The Oxford English Dictionary, Webster's Third New International Dictionary, Merriam-Webster's Collegiate Dictionary, and The American Heritage Dictionary of the English Language.

5 I am thinking, for example, of de Finetti's statement that "the only foundation which truly reflects the crucial elements of" the relationship between inductive reasoning and analogy is "intuitive (and therefore subjective)" (1985, p. 357). Also his 
statement that "we can only evaluate the probability according to our judgment" (1972, p. 188).

6 More precisely: 'The degree of belief in $H$ that is evidentially epistemically rational for a person whose total evidence is $E^{\prime}$ is just an obscure term for the inductive probability of $H$ given $E$.

7 I have discussed sentences like this with students in many classes and the students always believe such sentences are obviously true.

8 Franklin (2001, p. 289) also argues that P is false and furthermore inconsistent with other things that Ramsey says.

9 Obviously, this is not an accurate statement of Keynes's view, since Keynes clearly stated that the probabilities he was talking about were not degrees of belief.

10 In that paper I referred to the inductive probability of $H$ given $E$ as "the degree of belief in $H$ that is justified by $E$." For the reasons given in Section 2.3 of this paper, I now think that was an insufficiently clear way of identifying my explicandum.

\section{References}

Alston, W. P.: 1985, 'Concepts of Epistemic Justification'. The Monist 68(1), 57-89. Bacchus, F.: 1990, Representing and Reasoning with Probabilistic Knowledge. Cambridge, Mass.: MIT Press.

Bernardo, J. M. and A. F. M. Smith: 1994, Bayesian Theory. Chichester, England: Wiley. Paperback edition with corrections, 2000.

Carnap, R.: 1950, Logical Foundations of Probability. Chicago: University of Chicago Press. Second edition 1962.

de Finetti, B.: 1972, Probability, Induction and Statistics. London: Wiley.

de Finetti, B.: 1980, 'Probability: Beware of Falsifications!'. In (Kyburg and Smokler, 1980), pp. 193-224.

de Finetti, B.: 1985, 'Cambridge Probability Theorists'. The Manchester School of Economic and Social Studies 53(4), 348-363. Translation by Gianluigi Pelloni of an article written in the 1930s.

Franklin, J.: 2001, 'Resurrecting Logical Probability'. Erkenntnis 55, 277-305.

Gillies, D.: 2000, Philosophical Theories of Probability. London: Routledge.

Hacking, I.: 1975, The Emergence of Probability. Cambridge: Cambridge University Press.

Hacking, I.: 2001, An Introduction to Probability and Inductive Logic. Cambridge: Cambridge University Press.

Howson, C. and P. Urbach: 1993, Scientific Reasoning: The Bayesian Approach. Chicago: Open Court, second edition.

Keynes, J. M.: 1921, A Treatise on Probability. London: Macmillan. Reprinted with corrections 1948.

Kyburg, Jr., H. E. and H. E. Smokler (eds.): 1980, Studies in Subjective Probability. Huntington, New York: Krieger, 2nd edition.

Maher, P.: 1993, Betting on Theories. Cambridge: Cambridge University Press.

Maher, P.: 2004, 'Probability Captures the Logic of Scientific Confirmation'. In: C. R. Hitchcock (ed.): Contemporary Debates in Philosophy of Science. Oxford: Blackwell, pp. 69-93.

Ramsey, F. P.: 1926, 'Truth and Probability'. In (Kyburg and Smokler, 1980), pp. $25-52$. 
Roeper, P. and H. Leblanc: 1999, Probability Theory and Probability Logic. Toronto: University of Toronto Press.

Salmon, W. C.: 1967, The Foundations of Scientific Inference. Pittsburgh, PA: University of Pittsburgh Press.

Skyrms, B.: 1986, Choice and Chance: An Introduction to Inductive Logic. Belmont, CA: Wadsworth, third edition.

Suppe, F.: 1998, 'Operationalism'. In: E. Craig (ed.): Routledge Encyclopedia of Philosophy. London: Routledge.

van Fraassen, B. C.: 1989, Laws and Symmetry. Oxford: Oxford University Press. 